\title{
Classifying chest CT images as COVID-19 positive/negative using a convolutional neural network ensemble model and uniform experimental design method
}

\author{
Yao-Mei Chen ${ }^{1,2}$, Yenming J. Chen ${ }^{3}$, Wen-Hsien Ho $\mathrm{H}^{4,5^{*}}$ and Jinn-Tsong Tsai ${ }^{4,6^{*}}$ (D)
}

From International Conference on Biomedical Engineering Innovation 2019 Kaohsiung, Taiwan. 15-19 November 2019

${ }^{*}$ Correspondence:

whho@kmu.edu.tw;

jttsai@mail.nptu.edu.tw

${ }^{4}$ Department of Healthcare

Administration and Medical

Informatics, Kaohsiung

Medical University,

Kaohsiung 807, Taiwan

${ }^{6}$ Department of Computer

Science, National Pingtung

University, Pingtung 900,

Taiwan

Full list of author information

is available at the end of the article

\begin{abstract}
Background: To classify chest computed tomography (CT) images as positive or negative for coronavirus disease 2019 (COVID-19) quickly and accurately, researchers attempted to develop effective models by using medical images.

Results: A convolutional neural network (CNN) ensemble model was developed for classifying chest CT images as positive or negative for COVID-19. To classify chest CT images acquired from COVID-19 patients, the proposed COVID19-CNN ensemble model combines the use of multiple trained CNN models with a majority voting strategy. The CNN models were trained to classify chest CT images by transfer learning from well-known pre-trained CNN models and by applying their algorithm hyperparameters as appropriate. The combination of algorithm hyperparameters for a pre-trained CNN model was determined by uniform experimental design. The chest CT images (405 from COVID-19 patients and 397 from healthy patients) used for training and performance testing of the COVID19-CNN ensemble model were obtained from an earlier study by $\mathrm{Hu}$ in 2020. Experiments showed that, the COVID19-CNN ensemble model achieved $96.7 \%$ accuracy in classifying CT images as COVID-19 positive or negative, which was superior to the accuracies obtained by the individual trained CNN models. Other performance measures (i.e., precision, recall, specificity, and $\mathrm{F}_{1}$-score) obtained bythe COVID19-CNN ensemble model were higher than those obtained by individual trained CNN models.
\end{abstract}

Conclusions: The COVID19-CNN ensemble model had superior accuracy and excellent capability in classifying chest CT images as COVID-19 positive or negative.

Keywords: COVID-19, Chest computed tomography image, Convolutional neural network, Algorithm hyperparameter, Ensemble model author(s) and the source, provide a link to the Creative Commons licence, and indicate if changes were made. The images or other third party material in this article are included in the article's Creative Commons licence, unless indicated otherwise in a credit line to the material. If material is not included in the article's Creative Commons licence and your intended use is not permitted by statutory regulation or exceeds the permitted use, you will need to obtain permission directly from the copyright holder. To view a copy of this licence, visit http:// creativecommons.org/licenses/by/4.0/. The Creative Commons Public Domain Dedication waiver (http://creativecommons.org/publi cdomain/zero/1.0/) applies to the data made available in this article, unless otherwise stated in a credit line to the data. 


\section{Background}

The rapid spread of coronavirus disease 2019 (COVID-19) since the beginning of 2020 has often exceeded the capability of doctors and hospitals in many regions of the world. One effective tool for detecting COVID-19 is chest computed tomography (CT). Although a CT scan can be performed in several minutes, the time needed for a radiologist to review and classify the image is much longer. Therefore, tools for automatically detecting or diagnosing COVID-19 are extremely valuable and urgently needed.

\section{Literature review}

Gozes et al. [1] developed automated CT image analysis tools that used a Resnet-50 deep convolutional neural network $(\mathrm{CNN})$ to detect coronavirus and to quantify the burden on healthcare systems. The study reported that deep-learning image analysis of thoracic CT images achieved $98.2 \%$ sensitivity, $92.2 \%$ specificity, and 0.996 area under curve (AUC) in classifying results as positive or negative for coronavirus.Another COVID-19 diagnosis method developed in $\mathrm{Hu}$ et al. [2] used a CNN with a ShuffleNet-v2 backbone to distinguish between CT images of patients with and without COVID-19 infection. Their experimental results indicated that the diagnostic model was accurate not only in identifying COVID-19, but also in distinguishing between COVID-19 infections from other viral infections. Li et al. [3] developed a COVNet framework using Resnet-50 as the backbone that detected COVID-19 by using a neural network to extract visual features from volumetric chest CT exams. In independent testing, per-exam sensitivity in detecting COVID-19 was 90\% (114 of 127), and per-exam specificity was $96 \%$ (294 of 307). Shan et al. [4] developed a modified 3-D convolutional neural network that combines $\mathrm{V}$-Net with the bottle-neck structure for automatically segmenting and quantifying infected regions in CT scans of COVID-19 patients. Quantitative evaluations indicated that the system was highly accurate in automatically delineating infected regions. Song et al. [5] developed a CT diagnosis system that used a detailed relation extraction neural network to identify COVID-19 patients. According to their experimental results, the model identified COVID-19 infection with recall (sensitivity) of 0.93. Wang et al. [6] proposed a transfer learning neural network based on the Inception network that used chest CT images to screen for COVID-19. Internal validation tests revealed that the model had an overall accuracy of $89.5 \%$ with specificity of 0.88 and sensitivity of 0.87 . In the external testing dataset, the model showed a total accuracy of $79.3 \%$ with specificity of 0.83 and sensitivity of 0.67 . Xu et al. [7] established the Resnet-18 network with the location-attention mechanism that appeared promising for supplementary clinical use by frontline doctors in diagnosis and early screening of COVID-19 patients. Experiments performed using the benchmark dataset achieved 86.7\% accuracy in screening CT images for COVID-19. In Yang et al. [8], the multi-task learning and self-supervised learning method of COVID-19 diagnosis based on CT images of COVID-19 achieved an $\mathrm{F}_{1}$-score of 0.90 , an AUC of 0.98 , and an accuracy of 0.89 . According to the senior radiologist in that study, the models perform well enough for clinical use. According to the above literature on COVID-19 screening, most researchers have used a single model to classify chest $\mathrm{CT}$ images. Compared to an ensemble model in which classification is based on the results of the majority, however, a single model is more likely to 
make classification errors. Moreover, no studies have discussed how algorithm hyperparameters affect classification accuracy in a pre-trained $\mathrm{CNN}$ model. Therefore, further research is needed to improve classification accuracy.

\section{Motivation}

The motivation of this study was to establish an ensemble model that uses majority voting strategy to screen chest CT images for COVID-19. In a pre-trained CNN model, learning speed and quality are determined by algorithm hyperparameters that are set before the learning process begins. In subsequent training, different pre-trained $\mathrm{CNN}$ models may require different algorithm hyperparameters (e.g., optimizer, learning rate, and mini-batch size) to improve their classification accuracy [9]. The current study used uniform experimental design (UED) to generate the combination of algorithm hyperparameters for a pre-trained CNN model. The experiments showed that the COVID19$\mathrm{CNN}$ ensemble model had superior classification accuracy compared to a single model and excellent accuracy in classifying chest CT images as COVID-19 positive/negative.

\section{Problem description}

Chest CT and X-Ray images are critical practical tools for diagnosis of COVID-19, because they can be used relatively quickly and easily to detect pneumonia-like symptoms of COVID-19. A recent study concluded that screening lung CT images is the best method of early-stage COVID-19 diagnosis and concluded that CT should be the primary screening method [10]. The severe respiratory symptoms of COVID-19 result in relatively high ICU admission and mortality rates in these patients. Manifestations of COVID-19 in CT images differ from those of other viruses that cause pneumonia, e.g., influenza-A [7]. Therefore, CT images have an untapped potential use in COVID-19 diagnosis.

During a COVID-19 outbreak, overworked radiologists may have limited time to review CT scans. Additionally, radiologists in rural and/or under-developed areas may not be adequately trained to screen CT scans for an emerging disease such as COVID19. The considered problem was how to screen large numbers of chest CT images for COVID-19 efficiently and accurately. Since a CT showing evidence of COVID-19 is difficult to distinguish from a normal CT, machine learning may be a useful tool for assisting radiologists in screening CT images for COVID-19.

The key slices of chest $\mathrm{CT}$ with suspected lesions were extracted from DICOM files by professional radiologists. All chest CT images used in the experiments in this study had been published previously [11]. The CT images were divided into two classes: COVID19 and Normal. Figure 1 shows representative CT images in the two classes.

\section{Results}

The proposed COVID19-CNN ensemble model integrated multiple trained CNN models for classifying chest CT images as COVID-19 positive or negative. The pre-trained CNN models included VGG-19, Resnet-101, DenseNet-201, Inception-v3, and Inception-ResNet-v2. The chest CT images obtained from COVID-19 patients in Hu [11] were used for training and performance validation of pre-trained CNN models. The testing set of chest CT images from COVID-19 patients was used for performance evaluation of 


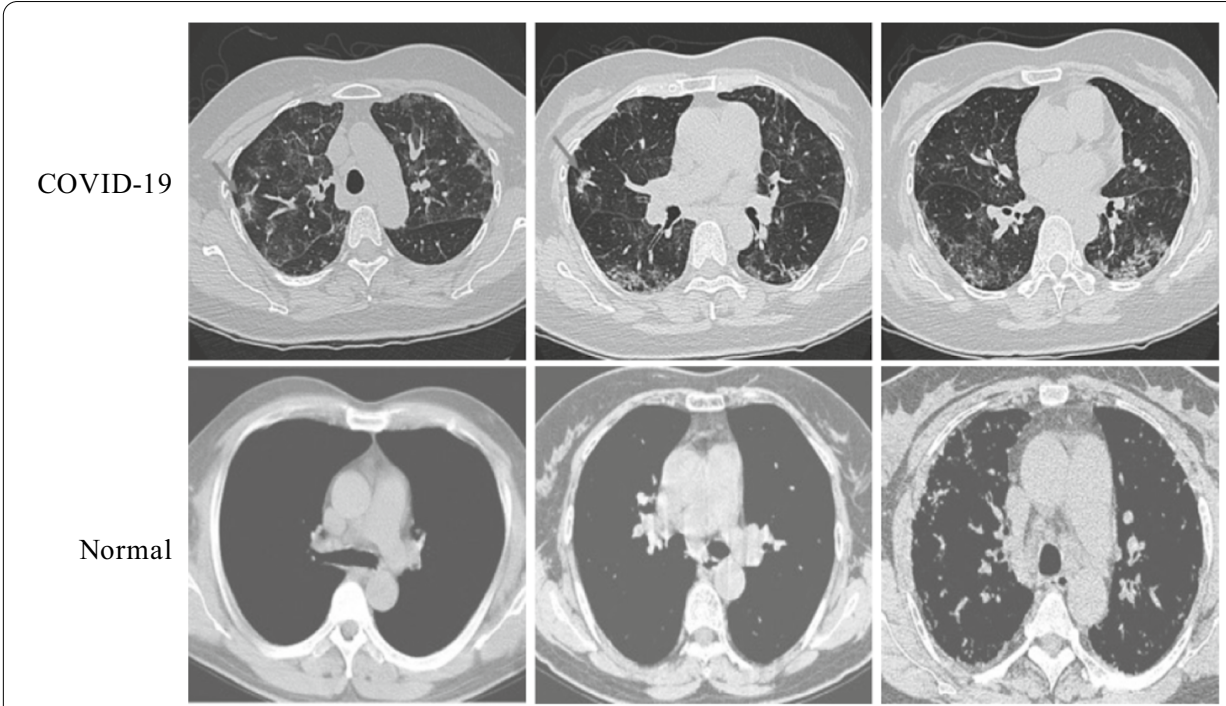

Fig. 1 Representative CT images in the COVID-19 and Normal classes

Table 1 Training, validation, and testing sets of chest CT images from COVID-19 patients

\begin{tabular}{lllll}
\hline Class & Training set & Validation set & Testing set & Total images \\
\hline COVID-19 & 309 & 50 & 46 & 405 \\
Normal & 303 & 49 & 45 & 397 \\
Total images & 612 & 99 & 91 & 802 \\
\hline
\end{tabular}

the COVID19-CNN ensemble model. The experimental environment was Matlab R2019 with its toolboxes developed by MathWorks, and GPU GTX-1080Ti-11G.

The experimental data for chest CT images from COVID-19 patients included a training set, a validation set, and a testing set. To maintain compatibility with the CNNbased architecture and the developed software, each CT image was processed as a $224 \times 224 \times 3$ image for the VGG-19, Resnet-101, and DenseNet-201 models or as a $299 \times 299 \times 3$ image for the Inception-v3 and Inception-ResNet-v2 models, where 3 is the number of color channels. Table 1 shows the training, validation, and testing sets of chest CT images from COVID-19 patients.

For training, different pre-trained $\mathrm{CNN}$ models require different algorithm hyperparameters that are set before the learning process begins. The algorithm hyperparameters for pre-trained CNN models in this study were 'Optimizer,' 'MiniBatchSize', 'MaxEpochs', and 'InitialLearnRate'. Optimizer was the training option. MiniBatchSize was a mini-batch at each iteration. MaxEpochs was the maximum number of training epochs. InitialLearnRate was an option for decreasing the learning rate during training.

The UED table of the minimum number of experiments for four factors is $U_{7}$. Tables 2 and 3 show the seven-level uniform layout and selection table used for $U_{7}\left(7^{6}\right)$, respectively. Table 4 shows that $U_{7}\left(7^{4}\right)$ was selected from four factors in Table 3 and was used to design the combinations of the four algorithm hyperparameters for the seven levels. The levels for the 'Optimizer' hyperparameter were 'adam (adaptive moment 
Table 2 Seven-level uniform layout of $U_{7}\left(7^{6}\right)$

\begin{tabular}{lllllll}
\hline Number of experiments & \multicolumn{2}{l}{ Number of columns } \\
\cline { 2 - 7 } & 1 & 2 & 3 & 4 & 5 & 6 \\
\hline 1 & 1 & 2 & 3 & 4 & 5 & 6 \\
2 & 2 & 4 & 6 & 1 & 3 & 5 \\
3 & 3 & 6 & 2 & 5 & 1 & 4 \\
4 & 4 & 1 & 5 & 2 & 6 & 3 \\
5 & 5 & 3 & 1 & 6 & 4 & 2 \\
7 & 6 & 5 & 4 & 3 & 2 & 1 \\
\hline
\end{tabular}

Table 3 Selection table used for $U_{7}\left(7^{6}\right)$

\begin{tabular}{ll}
\hline Number of factors & $\begin{array}{l}\text { Number } \\
\text { of } \\
\text { columns }\end{array}$ \\
\hline 2 & 13 \\
3 & 123 \\
4 & 1236 \\
\hline
\end{tabular}

Table 4 Seven-level uniform layout of $U_{7}\left(7^{4}\right)$ used to allocate four algorithm hyperparameters for seven levels

\begin{tabular}{lllll}
\hline $\begin{array}{l}\text { Number of } \\
\text { experiments }\end{array}$ & \multicolumn{4}{l}{ Algorithm hyperparameters } \\
\cline { 2 - 5 } & Optimizer & MiniBatchSize & MaxEpochs & InitialLearnRate \\
\hline 1 & 1 & 2 & 3 & 6 \\
2 & 2 & 4 & 6 & 5 \\
3 & 3 & 6 & 2 & 4 \\
4 & 4 & 1 & 5 & 3 \\
5 & 5 & 3 & 1 & 2 \\
6 & 6 & 5 & 4 & 1 \\
7 & 7 & 7 & 7 & 7 \\
\hline
\end{tabular}

estimation)' and 'sgdm (stochastic gradient descent with a momentum)'. The values for the 'MiniBatchSize' hyperparameter ranged from 10 to 40. The values for the 'MaxEpochs' hyperparameter ranged from 4 to 10 . The values for 'InitialLearnRate' hyperparameter were $10^{-4}, 10^{-5}$, and $10^{-6}$. Table 5 shows the level values of the four algorithm hyperparameters for a pre-trained $\mathrm{CNN}$ model. Table 6 shows the seven combinations of the four algorithm hyperparameters that combined the values in Tables 4 and 5 and were used in a pre-trained CNN model for classifying chest CT images as COVID-19 positive or negative.

According to the hyperparameter combination plan in Table 6, five independent experimental runs were performed for each hyperparameter combination. Table 7 shows the average correct rates and standard deviations (SDs) obtained by using each algorithm hyperparameter combination in Table 6 in five independent experimental runs when the VGG-19 was used to classify chest CT images as COVID-19 positive or 
Table 5 Level values of four algorithm hyperparameters for a pre-trained CNN model

\begin{tabular}{lllcl}
\hline $\begin{array}{l}\text { Number of } \\
\text { experiments }\end{array}$ & \multicolumn{4}{l}{ Algorithm hyperparameters } \\
\cline { 2 - 5 } & Optimizer & MiniBatchSize & MaxEpochs & InitialLearnRate \\
\hline 1 & adam & 10 & 4 & $10^{-4}$ \\
2 & sgdm & 15 & 5 & $10^{-5}$ \\
3 & adam & 20 & 6 & $10^{-6}$ \\
4 & sgdm & 25 & 7 & $10^{-4}$ \\
5 & adam & 30 & 8 & $10^{-5}$ \\
6 & sgdm & 35 & 9 & $10^{-6}$ \\
7 & adam & 40 & 10 & $10^{-4}$ \\
\hline
\end{tabular}

Table 6 Combinations of the four algorithm hyperparameters that combined the values in Tables 4 and 5 for a pre-trained CNN model

\begin{tabular}{lllll}
\hline $\begin{array}{l}\text { Number of } \\
\text { experiments }\end{array}$ & \multicolumn{4}{l}{ Algorithm hyperparameters } \\
\cline { 2 - 5 } & Optimizer & MiniBatchSize & MaxEpochs & InitialLearnRate \\
\hline 1 & adam & 15 & 6 & $10^{-6}$ \\
2 & sgdm & 25 & 9 & $10^{-5}$ \\
3 & adam & 35 & 5 & $10^{-4}$ \\
4 & sgdm & 10 & 8 & $10^{-6}$ \\
5 & adam & 20 & 4 & $10^{-5}$ \\
6 & sgdm & 30 & 7 & $10^{-4}$ \\
7 & adam & 40 & 10 & $10^{-4}$ \\
\hline
\end{tabular}

Table 7 Average correct rates and SDs in classifying chest CT images as COVID-19 positive or negative when VGG-19 and each algorithm hyperparameter combination in Table 6 were used in five independent experimental runs

\begin{tabular}{|c|c|c|c|c|c|c|c|c|}
\hline \multirow{2}{*}{$\begin{array}{l}\text { Model\# } \\
\text { Experiment } \\
\text { number }\end{array}$} & \multirow[t]{2}{*}{ Dataset } & \multicolumn{6}{|c|}{ Experimental runs } & \multirow[b]{2}{*}{ SD } \\
\hline & & 1 & 2 & 3 & 4 & 5 & Average & \\
\hline \multirow[t]{2}{*}{ VGG-19\#1 } & Training set & 0.8252 & 0.8415 & 0.8448 & 0.8399 & 0.8399 & 0.8383 & 0.00757 \\
\hline & Validation set & 0.8182 & 0.8182 & 0.8182 & 0.798 & 0.798 & 0.8101 & 0.01106 \\
\hline \multirow[t]{2}{*}{ VGG-19\#2 } & Training set & 0.8578 & 0.8611 & 0.8709 & 0.8513 & 0.8497 & 0.8582 & 0.00851 \\
\hline & Validation set & 0.8485 & 0.798 & 0.8283 & 0.8182 & 0.8182 & 0.8222 & 0.01835 \\
\hline \multirow[t]{2}{*}{ VGG-19\#3 } & Training set & 0.5621 & 0.5784 & 0.6405 & 0.5931 & 0.4951 & 0.5738 & 0.05285 \\
\hline & Validation set & 0.5859 & 0.6465 & 0.6566 & 0.6061 & 0.4949 & 0.5980 & 0.06447 \\
\hline \multirow[t]{2}{*}{ VGG-19\#4 } & Training set & 0.8072 & 0.8056 & 0.7974 & 0.8039 & 0.8088 & 0.8046 & 0.00441 \\
\hline & Validation set & 0.7879 & 0.7778 & 0.7879 & 0.7677 & 0.798 & 0.7839 & 0.01152 \\
\hline \multirow[t]{2}{*}{ VGG-19\#5 } & Training set & 0.915 & 0.9036 & 0.915 & 0.915 & 0.8938 & 0.9085 & 0.00958 \\
\hline & Validation set & 0.8384 & 0.8182 & 0.8081 & 0.8384 & 0.8182 & 0.8243 & 0.01355 \\
\hline \multirow[t]{2}{*}{ VGG-19\#6 } & Training set & 0.9346 & 0.9265 & 0.9265 & 0.9346 & 0.9314 & 0.9307 & 0.00407 \\
\hline & Validation set & 0.8485 & 0.8485 & 0.8485 & 0.8485 & 0.8586 & 0.8505 & 0.00452 \\
\hline \multirow[t]{2}{*}{ VGG-19\#7 } & Training set & 0.8775 & 0.7026 & 0.7435 & 0.8693 & 0.8415 & 0.8069 & 0.07901 \\
\hline & Validation set & 0.7374 & 0.7071 & 0.7475 & 0.8182 & 0.8081 & 0.7637 & 0.0477 \\
\hline
\end{tabular}




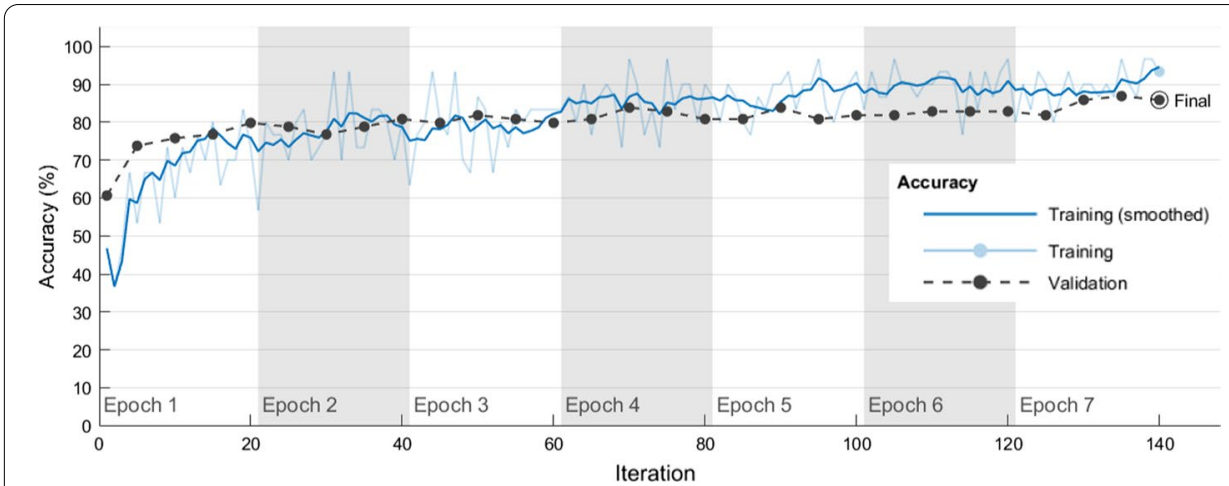

Fig. 2 Progressive improvement in accuracy of VGG-19\#6

Table 8 Average correct rates and SDs in classifying chest CT images as COVID-19 positive/negative when Resnet-101 and each algorithm hyperparameter combination in Table 6 were used in five independent experimental runs

\begin{tabular}{|c|c|c|c|c|c|c|c|c|}
\hline \multirow{2}{*}{$\begin{array}{l}\text { Model\# } \\
\text { experiment } \\
\text { number }\end{array}$} & \multirow[t]{2}{*}{ Dataset } & \multicolumn{6}{|c|}{ Experimental runs } & \multirow[b]{2}{*}{ SD } \\
\hline & & 1 & 2 & 3 & 4 & 5 & Average & \\
\hline \multirow[t]{2}{*}{ Resnet-101\#1 } & Training set & 0.7124 & 0.7059 & 0.7075 & 0.7042 & 0.7026 & 0.7065 & 0.00376 \\
\hline & Validation set & 0.6061 & 0.596 & 0.6263 & 0.6162 & 0.6263 & 0.6142 & 0.01317 \\
\hline \multirow[t]{2}{*}{ Resnet-101\#2 } & Training set & 0.7876 & 0.781 & 0.7876 & 0.7876 & 0.7892 & 0.7866 & 0.00321 \\
\hline & Validation set & 0.6566 & 0.6566 & 0.6566 & 0.6566 & 0.6566 & 0.6566 & 0 \\
\hline \multirow[t]{2}{*}{ Resnet-101\#3 } & Training set & 0.9804 & 0.9755 & 0.982 & 0.9853 & 0.982 & 0.9810 & 0.00357 \\
\hline & Validation set & 0.8788 & 0.8788 & 0.8788 & 0.8788 & 0.8788 & 0.8788 & 0 \\
\hline \multirow[t]{2}{*}{ Resnet-101\#4 } & Training set & 0.4951 & 0.5065 & 0.4951 & 0.4951 & 0.4951 & 0.4974 & 0.0051 \\
\hline & Validation set & 0.5051 & 0.4848 & 0.5051 & 0.5051 & 0.5051 & 0.5010 & 0.00908 \\
\hline \multirow[t]{2}{*}{ Resnet-101\#5 } & Training set & 0.9085 & 0.8987 & 0.9101 & 0.9101 & 0.9101 & 0.9075 & 0.00497 \\
\hline & Validation set & 0.7879 & 0.7273 & 0.7879 & 0.798 & 0.798 & 0.7798 & 0.02979 \\
\hline \multirow[t]{2}{*}{ Resnet-101\#6 } & Training set & 0.8366 & 0.8758 & 0.8317 & 0.835 & 0.835 & 0.8428 & 0.01852 \\
\hline & Validation set & 0.7475 & 0.7677 & 0.7475 & 0.7475 & 0.7475 & 0.7515 & 0.00903 \\
\hline \multirow[t]{2}{*}{ Resnet-101\#7 } & Training set & 0.9918 & 0.9869 & 0.9853 & 0.9804 & 0.9869 & 0.9863 & 0.00409 \\
\hline & Validation set & 0.8687 & 0.899 & 0.8889 & 0.8586 & 0.899 & 0.8828 & 0.01835 \\
\hline
\end{tabular}

negative in training and validation sets. The VGG-19\#6 model had average correct rates of $93.07 \%$ and $85.05 \%$ in the training and validation sets, respectively. The VGG-19\#6 also had small SDs of 0.00407 and 0.00452 in the training and validation sets, respectively. For classifying chest CT images as COVID-19 positive or negative, the best combination of the four algorithm hyperparameters in the VGG-19\#6 model was Optimizer of 'sgdm', MiniBatchSize of 30, MaxEpochs of 7, and InitialLearnRate of $10^{-4}$. Figure 2 shows how model training progressively improved accuracy in VGG-19\#6. Iterations per epoch were $20(\approx 612 / 30)$, which was the number of training images/MiniBatchSize. Maximum iterations were $140(=20 \times 7)$, which was iterations per epoch $\times$ MaxEpochs. The blue line shows the progressive improvement in accuracy for the training set, and the black line shows the progressive improvement in accuracy for the validation set.

Table 8 shows the average correct rates and SDs obtained by using each algorithm hyperparameter combination in Table 6 in five independent experimental runs when the 
Resnet-101 model was used to classify chest CT images as COVID-19 positive or negative in training and validation sets. The Resnet-101\#3 model had average correct rates of $98.10 \%$ and $87.88 \%$ in the training and validation sets, respectively. The Resnet-101\#3 model also had small SDs of 0.00357 and 0 in the training and validation sets, respectively. In the Resnet-101\#3 model, the hyperparameter combination with the high performance in classifying chest CT images as COVID-19 positive/negative was Optimizer of 'adam', MiniBatchSize of 35, MaxEpochs of 5, and InitialLearnRate of $10^{-4}$. The Resnet-101\#7 model had average correct rates of $98.63 \%$ and $88.28 \%$ in the training and validation sets, respectively. The Resnet-101\#7 model also had SDs of 0.00409 and 0.01835 in the training and validation sets, respectively. In the Resnet-101\#7 model, the hyperparameter combination with the best performance in classifying chest CT images as COVID-19 positive/negative was Optimizer of 'adam', MiniBatchSize of 40, MaxEpochs of 10, and InitialLearnRate of $10^{-4}$. Figure 3 shows how model training progressively improved accuracy in Resnet-101\#7. Iterations per epoch were 15( $\approx 612 / 40)$, which was the number of training images/MiniBatchSize. Maximum iterations were $150(=15 \times 10)$, which was iterations per epoch $\times$ MaxEpochs. The blue line shows the progressive improvement in accuracy for the training set, and the black line shows the progressive improvement in accuracy for the validation set.

Table 9 shows the average correct rates and SDs obtained for the training and validation sets when each algorithm hyperparameter combination in Table 6 was used in five independent experimental runs of the DenseNet-201 model to classify chest CT images as COVID-19 positive/negative. The DenseNet-201\#3 model had average correct rates of $98.89 \%$ and $87.27 \%$ in the training and validation sets, respectively. This DenseNet-201\#3 model also had small SDs of 0.00507 and 0.01532 in the training and validation sets, respectively. In the DenseNet-201\#3 model, the hyperparameter combination with the high performance in classifying chest CT images as COVID-19 positive/ negative was Optimizer of 'adam', MiniBatchSize of 35, MaxEpochs of 5, and InitialLearnRate of $10^{-4}$. The DenseNet-201\#7 model had average correct rates of $99.28 \%$ and $88.49 \%$ in the training and validation sets, respectively. The DenseNet-201\#7 model also had small SDs of 0.00469 and 0.0291 in the training and validation sets, respectively. In the DenseNet-201\#7 model, the hyperparameter combination with the best performance in classifying chest CT images as COVID-19 positive/negative was Optimizer of 'adam', MiniBatchSize of 40, MaxEpochs of 10, and InitialLearnRate of $10^{-4}$. Figure 4 shows

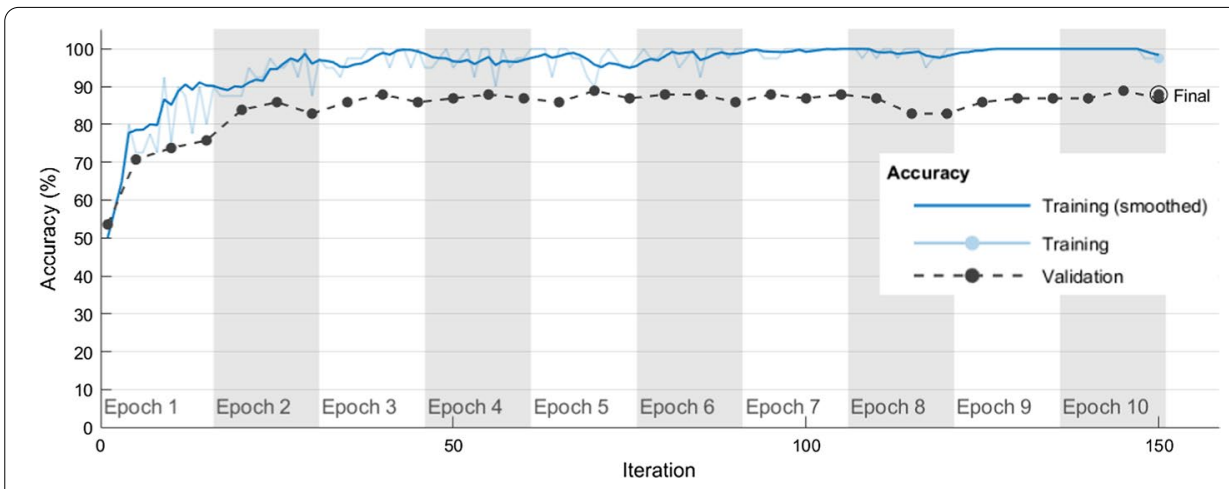

Fig. 3 Progressive improvement in accuracy of Resnet-101\#7 
Table 9 Average correct rates and SDs in classifying chest CT images as COVID-19 positive/negative when DenseNet-201 and each algorithm hyperparameter combination in Table 6 were used in five independent experimental runs

\begin{tabular}{|c|c|c|c|c|c|c|c|c|}
\hline \multirow[t]{2}{*}{ Model\# experiment number } & \multirow[t]{2}{*}{ Dataset } & \multicolumn{6}{|c|}{ Experimental runs } & \multirow[b]{2}{*}{ SD } \\
\hline & & 1 & 2 & 3 & 4 & 5 & Average & \\
\hline \multirow[t]{2}{*}{ DenseNet-201\#1 } & Training set & 0.7484 & 0.7484 & 0.7484 & 0.7533 & 0.7402 & 0.7477 & 0.00472 \\
\hline & Validation set & 0.6263 & 0.6263 & 0.6465 & 0.6364 & 0.6465 & 0.6364 & 0.0101 \\
\hline \multirow[t]{2}{*}{ DenseNet-201\#2 } & Training set & 0.7745 & 0.7745 & 0.781 & 0.7859 & 0.7859 & 0.7804 & 0.00571 \\
\hline & Validation set & 0.6667 & 0.6667 & 0.6667 & 0.6566 & 0.6768 & 0.6667 & 0.00714 \\
\hline \multirow[t]{2}{*}{ DenseNet-201\#3 } & Training set & 0.9902 & 0.9853 & 0.9886 & 0.9967 & 0.9837 & 0.9889 & 0.00507 \\
\hline & Validation set & 0.8788 & 0.8889 & 0.8788 & 0.8485 & 0.8687 & 0.8727 & 0.01532 \\
\hline \multirow[t]{2}{*}{ DenseNet-201\#4 } & Training set & 0.598 & 0.598 & 0.6062 & 0.6078 & 0.6013 & 0.6023 & 0.00457 \\
\hline & Validation set & 0.4949 & 0.4949 & 0.4848 & 0.4949 & 0.5051 & 0.4949 & 0.00718 \\
\hline \multirow[t]{2}{*}{ DenseNet-201\#5 } & Training set & 0.9265 & 0.9281 & 0.9281 & 0.9248 & 0.9167 & 0.9248 & 0.00475 \\
\hline & Validation set & 0.7677 & 0.7677 & 0.8182 & 0.7778 & 0.7879 & 0.7839 & 0.02094 \\
\hline \multirow[t]{2}{*}{ DenseNet-201\#6 } & Training set & 0.9183 & 0.9183 & 0.9134 & 0.9118 & 0.9118 & 0.9147 & 0.00333 \\
\hline & Validation set & 0.7677 & 0.7677 & 0.7677 & 0.8182 & 0.7576 & 0.7758 & 0.02411 \\
\hline \multirow[t]{2}{*}{ DenseNet-201\#7 } & Training set & 0.9951 & 0.9967 & 0.9869 & 0.9886 & 0.9967 & 0.9928 & 0.00469 \\
\hline & Validation set & 0.899 & 0.9293 & 0.8687 & 0.8586 & 0.8687 & 0.8849 & 0.0291 \\
\hline
\end{tabular}

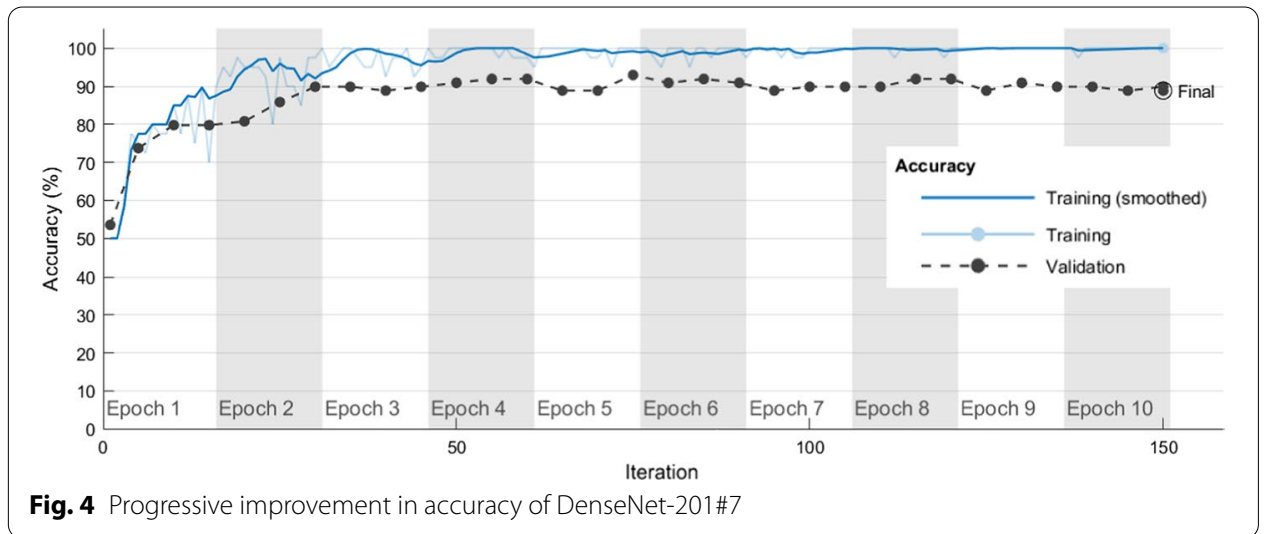

how model training progressively improved accuracy in DenseNet-201\#7. Iterations per epoch were $15(\approx 612 / 40)$, which was the number of training images/MiniBatchSize. Maximum iterations were $150(=15 \times 10)$, which was iterations per epoch $\times$ MaxEpochs. The blue line shows the progressive improvement in accuracy for the training set, and the black line shows the progressive improvement in accuracy for the validation set.

Table 10 shows the average correct rates and SDs obtained when each algorithm hyperparameter combination in Table 6 was used in five independent experimental runs of Inception-v3 to classify chest CT images as COVID-19 positive/negative in the training and validation sets. The Inception-v3\#7 model had average correct rates of $98.89 \%$ and $86.67 \%$ in the training and validation sets, respectively. The Inception-v3\#7 also had small SDs of 0.00355 and 0.01317 in the training and validation sets, respectively. In the Inception-v3\#7 model, the hyperparameter combination with the best performance in classifying chest CT images as COVID-19 positive/negative was Optimizer of 'adam, MiniBatchSize of 40, MaxEpochs of 10, and InitialLearnRate of $10^{-4}$. Figure 5 shows how 
Table 10 Average correct rates and SDs in classifying chest CT images as COVID-19 positive/ negative when Inception-v3 and each algorithm hyperparameter combination in Table 6 were used in five independent experimental runs

\begin{tabular}{|c|c|c|c|c|c|c|c|c|}
\hline \multirow{2}{*}{$\begin{array}{l}\text { Model\# } \\
\text { experiment } \\
\text { number }\end{array}$} & \multirow[t]{2}{*}{ Dataset } & \multicolumn{6}{|c|}{ Experimental runs } & \multirow[b]{2}{*}{ SD } \\
\hline & & 1 & 2 & 3 & 4 & 5 & Average & \\
\hline \multirow[t]{2}{*}{ Inception-v3\#1 } & Training set & 0.7418 & 0.7533 & 0.75 & 0.75 & 0.7484 & 0.7487 & 0.00425 \\
\hline & Validation set & 0.7172 & 0.7475 & 0.7576 & 0.7374 & 0.7374 & 0.7394 & 0.01498 \\
\hline \multirow[t]{2}{*}{ Inception-v3\#2 } & Training set & 0.6814 & 0.6699 & 0.683 & 0.6716 & 0.6716 & 0.6755 & 0.00618 \\
\hline & Validation set & 0.7172 & 0.7071 & 0.7172 & 0.7071 & 0.7071 & 0.7111 & 0.00553 \\
\hline \multirow[t]{2}{*}{ Inception-v3\#3 } & Training set & 0.9869 & 0.9869 & 0.9853 & 0.9869 & 0.982 & 0.9856 & 0.00213 \\
\hline & Validation set & 0.8283 & 0.8485 & 0.8687 & 0.8485 & 0.8485 & 0.8485 & 0.01428 \\
\hline \multirow[t]{2}{*}{ Inception-v3\#4 } & Training set & 0.5163 & 0.5196 & 0.5229 & 0.5147 & 0.5147 & 0.5176 & 0.00356 \\
\hline & Validation set & 0.5556 & 0.5657 & 0.5859 & 0.5859 & 0.5859 & 0.5758 & 0.01428 \\
\hline \multirow[t]{2}{*}{ Inception-v3\#5 } & Training set & 0.9118 & 0.8938 & 0.902 & 0.9003 & 0.9003 & 0.9016 & 0.00649 \\
\hline & Validation set & 0.8182 & 0.8182 & 0.8283 & 0.7879 & 0.798 & 0.8101 & 0.0166 \\
\hline \multirow[t]{2}{*}{ Inception-v3\#6 } & Training set & 0.8448 & 0.817 & 0.8301 & 0.8513 & 0.8513 & 0.8389 & 0.01499 \\
\hline & Validation set & 0.798 & 0.7677 & 0.7677 & 0.7879 & 0.7879 & 0.7818 & 0.01355 \\
\hline \multirow[t]{2}{*}{ Inception-v3\#7 } & Training set & 0.9869 & 0.9869 & 0.9918 & 0.9935 & 0.9853 & 0.9889 & 0.00355 \\
\hline & Validation set & 0.8788 & 0.8788 & 0.8586 & 0.8687 & 0.8485 & 0.8667 & 0.01317 \\
\hline
\end{tabular}

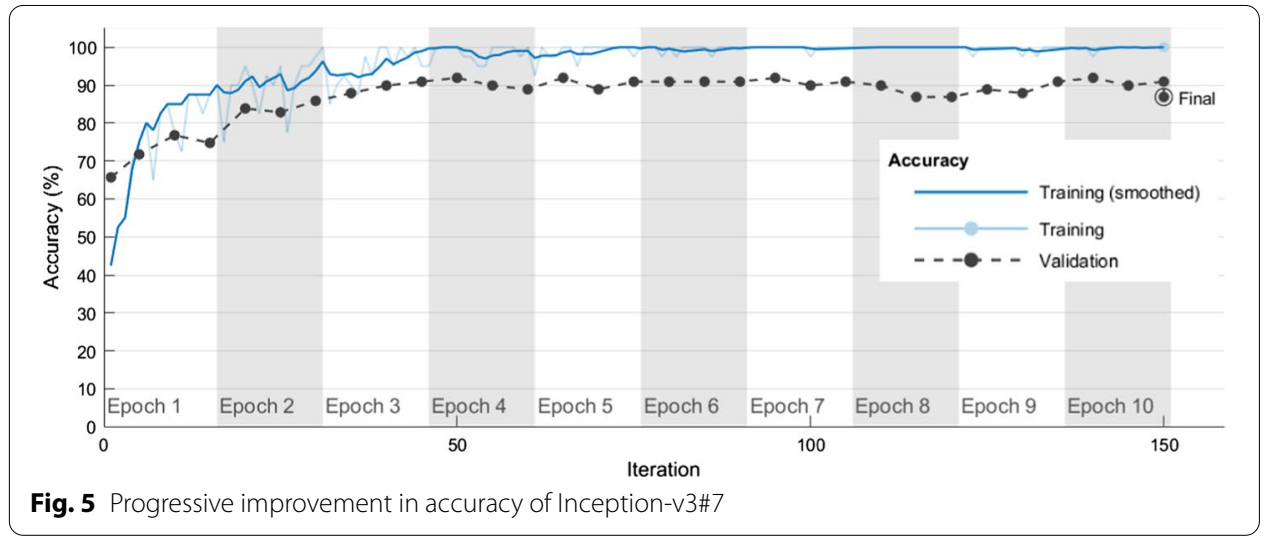

model training progressively improved accuracy in Inception-v3\#7. Iterations per epoch were $15(\approx 612 / 40)$, which was the number of training images/MiniBatchSize. Maximum iterations were $150(=15 \times 10)$, which was iterations per epoch $\times$ MaxEpochs. The blue line shows the progressive improvement in accuracy for the training set, and the black line shows the progressive improvement in accuracy for the validation set.

Table 11 shows the average correct rates and SDs obtained when each algorithm hyperparameter combination in Table 6 was used in five independent experimental runs of Inception-ResNet-v2 to classify chest CT images as COVID-19 positive/negative in the training and validation sets. The Inception-ResNet-v2\#3 model had average correct rates of $98.20 \%$ and $88.08 \%$ in the training and validation sets, respectively. The Inception-ResNet-v2\#3 model also had small SDs of 0.00475 and 0.01807 in the training and validation sets, respectively. In the Inception-ResNet-v2\#3 model, the hyperparameter combination with the high performance in classifying chest CT images as COVID-19 
Table 11 Average correct rates and SDs in classifying chest CT images as COVID-19 positive/ negative when Inception-ResNet-v2 and each algorithm hyperparameter combination in Table 6 were used in five independent experimental runs

\begin{tabular}{|c|c|c|c|c|c|c|c|c|}
\hline \multirow[t]{2}{*}{ Model\# experiment number } & \multirow[t]{2}{*}{ Dataset } & \multicolumn{6}{|c|}{ Experimental runs } & \multirow[b]{2}{*}{ SD } \\
\hline & & 1 & 2 & 3 & 4 & 5 & Average & \\
\hline \multirow[t]{2}{*}{ Inception-ResNet-v2\#1 } & Training set & 0.7092 & 0.7157 & 0.7271 & 0.7157 & 0.7141 & 0.7164 & 0.00657 \\
\hline & Validation set & 0.6061 & 0.6061 & 0.6162 & 0.6061 & 0.6061 & 0.6081 & 0.00452 \\
\hline \multirow[t]{2}{*}{ Inception-ResNet-v2\#2 } & Training set & 0.6471 & 0.6503 & 0.6454 & 0.6487 & 0.6307 & 0.6444 & 0.00789 \\
\hline & Validation set & 0.5253 & 0.5051 & 0.5253 & 0.5051 & 0.5051 & 0.5132 & 0.01106 \\
\hline \multirow{2}{*}{ Inception-ResNet-v2\#3 } & Training set & 0.9755 & 0.9837 & 0.9853 & 0.9869 & 0.9788 & 0.9820 & 0.00475 \\
\hline & Validation set & 0.899 & 0.8687 & 0.899 & 0.8788 & 0.8586 & 0.8808 & 0.01807 \\
\hline \multirow[t]{2}{*}{ Inception-ResNet-v2\#4 } & Training set & 0.5114 & 0.5049 & 0.5098 & 0.5098 & 0.5033 & 0.5078 & 0.00352 \\
\hline & Validation set & 0.4848 & 0.4848 & 0.4848 & 0.4848 & 0.5051 & 0.4889 & 0.00908 \\
\hline \multirow[t]{2}{*}{ Inception-ResNet-v2\#5 } & Training set & 0.8971 & 0.902 & 0.9069 & 0.9003 & 0.9069 & 0.9026 & 0.00427 \\
\hline & Validation set & 0.7677 & 0.7778 & 0.7475 & 0.7677 & 0.7879 & 0.7697 & 0.01498 \\
\hline \multirow[t]{2}{*}{ Inception-ResNet-v2\#6 } & Training set & 0.7958 & 0.7876 & 0.8056 & 0.7876 & 0.781 & 0.7915 & 0.00946 \\
\hline & Validation set & 0.6768 & 0.7172 & 0.697 & 0.7172 & 0.6566 & 0.6930 & 0.02634 \\
\hline \multirow[t]{2}{*}{ Inception-ResNet-v2\#7 } & Training set & 0.9886 & 0.9755 & 0.9918 & 0.9869 & 0.9902 & 0.9866 & 0.00647 \\
\hline & Validation set & 0.9192 & 0.9192 & 0.899 & 0.899 & 0.9091 & 0.9091 & 0.0101 \\
\hline
\end{tabular}

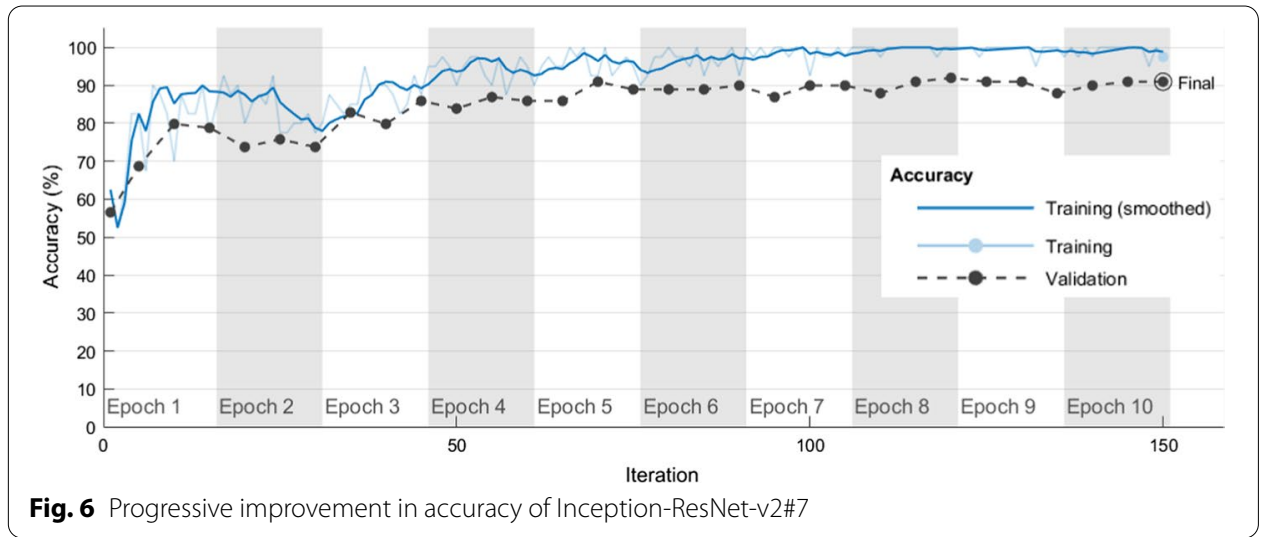

positive/negative was Optimizer of 'adam', MiniBatchSize of 35, MaxEpochs of 5, and InitialLearnRate of $10^{-4}$. The Inception-ResNet-v2\#7 model had average correct rates of $98.66 \%$ and $90.91 \%$ in the training and validation sets, respectively. The InceptionResNet-v2\#7 model also had small SDs of 0.00647 and 0.0101 in the training and validation sets, respectively. The hyperparameter combination with the best performance in classifying chest CT images as COVID-19 positive/negative was Optimizer of 'adam', MiniBatchSize of 40, MaxEpochs of 10, and InitialLearnRate of $10^{-4}$. Figure 6 shows how model training progressively improved accuracy in Inception-ResNet-v2\#7. Iterations per epoch were $15(\approx 612 / 40)$, which was the number of training images/MiniBatchSize. Maximum iterations were $150(=15 \times 10)$, which was iterations per epoch $\times$ MaxEpochs. The blue line shows the progressive improvement in accuracy for the training set, and the black line shows the progressive improvement in accuracy for the validation set.

According to the results of the thirty-five trained CNNs (shown in Tables 7, 8, 9, 10 and 11), the trained $\mathrm{CNN}$ shows that the average correct rates on the training set are high, 
and the average correct rates on the verification set are high. Table 12 shows the high classification accuracy obtained by the Resnet-101\#3, Resnet-101\#7, DenseNet-201\#3, DenseNet-201\#7, Inception-v3\#7, Inception-ResNet-v2\#3, and Inception-ResNet-v2\#7 models. The SDs on the training set of the seven models are between 0.003 and 0.0065 , indicating that the classification ability of the seven models is quite stable. The seven models for the validation set had average correct rates exceeding 0.86 , though the average correct rate on the training set is $10 \%$ higher than that on the validation set. Therefore, the seven models were selected for inclusion in the ensemble model for classifying chest CT images as COVID-19 positive/negative.

The COVID19-CNN ensemble model, which combined Resnet-101\#3, Resnet-101\#7, DenseNet-201\#3, DenseNet-201\#7, Inception-v3\#7, Inception-ResNet-v2\#3, and Inception-ResNet-v2\#7, used a majority voting strategy to classify chest CT images as COVID-19 positive/negative. An image classified as COVID-19 positive by most models was considered a COVID-19 image, and an image classified as COVID-19 negative by most models was considered a Normal image. The COVID19-CNN ensemble model aggregated the results of the majority voting strategy.

The accuracy metric was used to measure the performance of the Resnet-101\#3, Resnet-101\#7, DenseNet-201\#3, DenseNet-201\#7, Inception-v3\#7, Inception-ResNetv2\#3, Inception-ResNet-v2\#7, and COVID19-CNN models. Precision, recall, specificity, and $\mathrm{F}_{1}$-score were further used to validate classification performance. The results were depicted by creating a confusion matrix of the predicted labels versus the true labels for the respective classes. Table 13 shows the confusion matrix used for comparisons of COVID-19 positive and negative images in the Resnet-101\#3, Resnet-101\#7, DenseNet-201\#3, DenseNet-201\#7, Inception-v3\#7, Inception-ResNet-v2\#3, InceptionResNet-v2\#7, and COVID19-CNN models for the testing set.

Based on the data in Tables 13 and 14 shows the classifier accuracy, precision, recall, specificity, and $\mathrm{F}_{1}$-score obtained by the different trained CNN models and COVID19$\mathrm{CNN}$ ensemble models. When the testing set was used in the COVID19-CNN ensemble

Table 12 High accuracy of models obtained by Resnet-101\#3, Resnet-101\#7, DenseNet-201\#3, DenseNet-201\#7, Inception-v3\#7, Inception-ResNet-v2\#3, and Inception-ResNet-v2\#7

\begin{tabular}{llll}
\hline Model & Dataset & Average & SD \\
\hline Resnet-101\#3 & Training set & 0.9810 & 0.00357 \\
Resnet-101\#7 & Validation set & 0.8788 & 0 \\
& Training set & 0.9863 & 0.00409 \\
DenseNet-201\#3 & Validation set & 0.8828 & 0.01835 \\
& Training set & 0.9889 & 0.00507 \\
DenseNet-201\#7 & Validation set & 0.8727 & 0.01532 \\
Inception-v3\#7 & Training set & 0.9928 & 0.00469 \\
& Validation set & 0.8849 & 0.0291 \\
Inception-ResNet-v2\#3 & Training set & 0.9889 & 0.00355 \\
Inception-ResNet-v2\#7 & Validation set & 0.8667 & 0.01317 \\
& Training set & 0.9820 & 0.00475 \\
& Validation set & 0.8808 & 0.01807 \\
& Training set & 0.9866 & 0.00647 \\
\hline
\end{tabular}


Table 13 Confusion matrix for COVID-19 and Normal images obtained by the different trained CNN models and the COVID19-CNN ensemble model for the testing set

\begin{tabular}{|c|c|c|c|c|}
\hline \multirow[t]{2}{*}{ Model } & & & \multicolumn{2}{|l|}{ True Labels } \\
\hline & & & COVID-19 & Normal \\
\hline \multirow[t]{2}{*}{ Resnet-101\#3 } & Predicted & COVID-19 & 42 & 4 \\
\hline & Labels & Normal & 4 & 41 \\
\hline \multirow[t]{2}{*}{ Resnet-101\#7 } & Predicted & COVID-19 & 43 & 3 \\
\hline & Labels & Normal & 3 & 42 \\
\hline \multirow[t]{2}{*}{ DenseNet-201\#3 } & Predicted & COVID-19 & 43 & 2 \\
\hline & Labels & Normal & 3 & 43 \\
\hline \multirow{2}{*}{ DenseNet-201\#7 } & Predicted & COVID-19 & 45 & 7 \\
\hline & Labels & Normal & 1 & 38 \\
\hline \multirow[t]{2}{*}{ Inception-v3\#7 } & Predicted & COVID-19 & 43 & 5 \\
\hline & Labels & Normal & 3 & 40 \\
\hline \multirow[t]{2}{*}{ Inception-ResNet-v2\#3 } & Predicted & COVID-19 & 45 & 3 \\
\hline & Labels & Normal & 1 & 42 \\
\hline \multirow[t]{2}{*}{ Inception-ResNet-v2\#7 } & Predicted & COVID-19 & 44 & 4 \\
\hline & Labels & Normal & 2 & 41 \\
\hline \multirow[t]{2}{*}{ COVID19-CNN } & Predicted & COVID-19 & 45 & 2 \\
\hline & Labels & Normal & 1 & 43 \\
\hline
\end{tabular}

Table 14 Classifier accuracy, precision, recall, specificity, and $F_{1}$-score obtained by different trained CNN models and by the COVID19-CNN ensemble model for the testing set

\begin{tabular}{llllll}
\hline Model & Accuracy & Precision & Recall & Specificity & F $_{1}$-score \\
\hline Resnet-101\#3 & 0.912 & 0.913 & 0.913 & 0.911 & 0.913 \\
Resnet-101\#7 & 0.934 & 0.935 & 0.935 & 0.933 & 0.935 \\
DenseNet-201\#3 & 0.945 & 0.956 & 0.935 & 0.956 & 0.945 \\
DenseNet-201\#7 & 0.912 & 0.865 & 0.978 & 0.844 & 0.918 \\
Inception-v3\#7 & 0.912 & 0.896 & 0.935 & 0.889 & 0.915 \\
Inception-ResNet-V2\#3 & 0.956 & 0.938 & 0.978 & 0.933 & 0.957 \\
Inception-ResNet-V2\#7 & 0.934 & 0.917 & 0.957 & 0.911 & 0.936 \\
COVID19-CNN & 0.967 & 0.957 & 0.978 & 0.956 & 0.968
\end{tabular}

model, the accuracy was 0.967 , which was superior to the accuracies obtained by the different trained $\mathrm{CNN}$ models. Other performance measures (i.e., precision, recall, specificity, and $\mathrm{F}_{1}$-score) obtained bythe COVID19-CNN ensemble model were higher than those obtained by the different trained CNN models. That is, the COVID19-CNN ensemble model had superior accuracy in classifying chest CT images as COVID-19 positive/negative.

For the testing set of chest CT images from COVID-19 patients, the number of COVID-19 images ranged from 1 to 46, and the number of Normal images ranged from 47 to 91 . Table 15 shows the numbers of images classified incorrectly by Resnet-101\#3, Resnet-101\#7, DenseNet-201\#3, DenseNet-201\#7, Inception-v3\#7, Inception-ResNetv2\#3, Inception-ResNet-v2\#7, and the COVID19-CNN ensemble model in the testing set. Eight classification errors occurred in the Resnet-101\#3, DenseNet-201\#7, and 
Table 15 Image numbers classified as wrong obtained by different trained CNN models and the COVID19-CNN ensemble model for the testing set

\begin{tabular}{ll}
\hline Model & Numbers \\
\hline Resnet-101\#3 & $11,25,34,46,64,70,78,83$ \\
Resnet-101\#7 & $25,28,46,58,70,78$ \\
DenseNet-201\#3 & $11,28,46,64,70$ \\
DenseNet-201\#7 & $11,60,64,70,75,78,80,81$ \\
Inception-v3\#7 & $1,29,46,52,70,77,78,90$ \\
Inception-ResNet-v2\#3 & $46,53,60,70$ \\
Inception-ResNet-v2\#7 & $1,28,53,61,70,83$ \\
COVID19-CNN & $46,70,78$ \\
\hline
\end{tabular}

Inception-v3\#7 models. Six classification errors occurred in the Resnet-101\#7 and Inception-ResNet-v2\#7 models. Five classification errors occurred in the DenseNet-201\#3 models. Four classification errors occurred in the Inception-ResNet-v2\#3 model. Image no. 46 was a COVID-19 image but was incorrectly classified as a Normal image by five models (i.e., Resnet-101\#3, Resnet-101\#7, DenseNet-201\#3, Inception-v3\#7, and Inception-ResNet-v2\#3). Image no. 70 was a Normal image but was incorrectly classified as a COVID-19 image by seven models (i.e., Resnet-101\#3, Resnet-101\#7, DenseNet-201\#3, DenseNet-201\#7, Inception-v3\#7, Inception-ResNet-v2\#3, and Inception-ResNet-v2\#7). Image no. 78 was a Normal image but was incorrectly classified as a COVID-19 image by four models (i.e., Resnet-101\#3, Resnet-101\#7, DenseNet-201\#7, and Inception-v3\#7). Therefore, the total number of classification errors made by the COVID19-CNN ensemble model using majority voting strategy was three. That is, the COVID19-CNN ensemble model had excellent capability in classifying chest CT images as COVID-19 positive/ negative. Notably, image numbers 70 and 46 were incorrectly classified by seven and five models, respectively, and should be reviewed by a radiologist.

\section{Discussion}

This study found that setting an appropriate combination of algorithm hyperparameters for a pre-trained $\mathrm{CNN}$ model was very important for accurately classifying chest CT images as COVID-19 positive or negative. In the VGG-19\#6 model, for example, the appropriate combination of the four algorithm hyperparameters for classifying CT images was Optimizer of 'sgdm', MiniBatchSize of 30, MaxEpochs of 7, and InitialLearnRate of $10^{-4}$. In the Resnet-101\#7, DenseNet-201\#7, Inception-v3\#7, and Inception-ResNet-v2\#7 models, the appropriate combination was Optimizer of 'adam, MiniBatchSize of 40, MaxEpochs of 10, and InitialLearnRate of $10^{-4}$. In Resnet-101\#3, DenseNet-201\#3, and Inception-ResNet-v2\#3, the appropriate combination was Optimizer of 'adam', MiniBatchSize of 35, MaxEpochs of 5, and InitialLearnRate of $10^{-4}$. Based on this study, it can be seen that a poor combination of algorithm hyperparameters for a pre-trained $\mathrm{CNN}$ model cannot get high accuracy in classifying chest CT images as COVID-19 positive/negative.

Although, from the novelty perspective, the contribution may be a relatively minor innovation, the COVID19-CNN ensemble model provided increased accuracy by applying a majority voting strategy and an appropriate combination of algorithm 
hyperparameters obtained by uniform experimental design can obtain high classification accuracy. Different trained CNN models had different results in classification of chest CT images as COVID-19 positive/negative, but the COVID19-CNN ensemble model used a majority voting mechanism to aggregate the results. Just like classifying chest $\mathrm{CT}$ images as COVID-19 positive/negative, the final classification results are determined by the opinions of most radiologists.

\section{Conclusions}

This COVID19-CNN ensemble model proposed in this study effectively classified chest CT images as COVID-19 positive/negative. The main contributions of this study are the confirmation that the ensemble model provides increased accuracy by applying a majority voting strategy and the confirmation that an appropriate combination of algorithm hyperparameters can obtain high classification accuracy. Additionally, the image number of misclassifications can be found by the COVID19-CNN ensemble model when classifying chest CT images as COVID-19 positive/negative. When the COVID19$\mathrm{CNN}$ ensemble model was used to classify CT images from the testing set of images as COVID-19 positive or negative, accuracy was $96.7 \%$, which was superior to the accuracies obtained by Resnet-101\#3 (91.2\% accuracy), Resnet-101\#7 (93.4\% accuracy), DenseNet-201\#3 (94.5\% accuracy), DenseNet-201\#7 (91.2\% accuracy), Inception-v3\#7 (91.2\% accuracy), Inception-ResNet-v2\#3 (95.6\% accuracy), and Inception-ResNetv2\#7 (93.4\% accuracy). Other performance measures obtained for the COVID19-CNN ensemble model (i.e., $95.7 \%$ precision, $97.8 \%$ recall, $95.6 \%$ specificity, and $96.8 \% \mathrm{~F}_{1}$-score) were also superior to those obtained by the different trained CNN models. That is, the COVID19-CNN ensemble model has excellent capability in classifying chest CT images as COVID-19 positive/negative.

\section{Methods}

The research procedure was collecting data and processing chest CT images from COVID-19 patients, selecting multiple pre-trained CNN models for transfer learning, using UED to set algorithm hyperparameters for pre-trained CNN models, using multiple pre-trained CNN models to screen chest CT images for COVID-19, comparing classification performance among the trained CNN models, selecting the high accurate CNN models for further use in an ensemble model and, finally, comparing classification performance in the trained $\mathrm{CNN}$ models. The detailed steps were as follows.

\section{Collecting data and processing chest CT images from COVID-19 patients}

The chest $\mathrm{CT}$ images from COVID-19 patients in $\mathrm{Hu}$ [11] were divided into a training set, a validation set, and a testing set. The training set had 612 chest CT images, including 309 images from COVID-19 patients and 303 images for the normal condition. The validation set had 99 chest $\mathrm{CT}$ images, including 50 images from COVID-19 patients and 49 images for the normal condition. The testing set used for network performance benchmarking contained 91 chest CT images, including 46 images from COVID-19 patients and 45 images for the normal condition. To maintain compatibility with the CNN-based architecture and the developed software, each CT image was processed 
as a $224 \times 224 \times 3$ image or as a $299 \times 299 \times 3$ image, where 3 is the number of color channels.

\section{Selecting multiple pre-trained CNN models for transfer learning}

Transfer learning is a machine learning approach in which a model developed for a task is reused as the starting point for a model developed for another task. In transfer learning, a pre-trained CNN model is used to construct a predictive model. Thus, the first step is selecting a pre-trained $\mathrm{CNN}$ model from available models. The second step is reusing the pre-trained $\mathrm{CNN}$ model. The third step is tuning the pre-trained $\mathrm{CNN}$ model for a new task. Depending on the input-output pair data available for the new task, the researcher may consider further modification or refinement of the pre-trained CNN model. Transfer learning in a CNN model with pre-training is typically much faster than that in a CNN model without pre-training.

The widely used commercial software program Matlab R2019 by MathWorks has been validated as effective for pre-training $\mathrm{CNN}$ models for deep learning. Most pre-trained CNN models were trained with a subset of the ImageNet database [12] used in the ImageNet Large-Scale Visual Recognition Challenge [13]. After training on more than 1 million images, the pre-trained CNN models could classify images into 1000 object categories, e.g., keyboard, coffee mug, pencil, and various animals. The most important characteristics of pre-trained CNN models are network accuracy, speed, and size. Choosing a pre-trained network is generally a tradeoff between these characteristics. The classification accuracy on the ImageNet validation set is the most common way to measure the accuracy of networks trained on ImageNet. Networks that are accurate on ImageNet are also often accurate when you apply them to other natural image data sets using transfer learning or feature extraction.

According to comparing the ImageNet validation accuracy with the network accuracy, speed, and size, the pre-trained CNNs used to classify chest CT images were VGG-19, Resnet-101, DenseNet-201, Inception-v3, and Inception-ResNet-v2.

The VGG-19 [14], Resnet-101 [15], and DenseNet-201 [16] CNNs have 19 layers, 101 layers, and 201 layers, respectively, and have been trained on more than 1 million images from the ImageNet database. As a result, these CNNs have learned rich feature representations for a wide range of images and can classify images into 1000 object categories. The image input size for these CNNs is $224 \times 224 \times 3$.

The 48-layer Inception-v3 [17] and the 164-layer Inception-ResNet-v2 [18] CNNs have been trained on more than 1 million images from the ImageNet database and can classify images into 1000 object categories. The image input size for these CNNs is $299 \times 299 \times 3$

\section{Using UED to design algorithm hyperparameters for pre-trained CNN models}

The UED method developed by Wang and Fang [19-21] used space filling designs to construct a set of experimental points uniformly scattered in a continuous design parameter space. Because UED only considers uniform dispersion and not comparable orderliness, UED minimizes the number of experiments needed to acquire all available information. 
Selecting appropriate algorithm hyperparameters for a pre-trained CNN model was essential for accurate screening of chest CT images for COVID-19. In this study, the algorithm hyperparameters for a pre-trained CNN model were Optimizer, MiniBatchSize, MaxEpochs, and InitialLearnRate. The combinations of algorithm hyperparameters obtained by UED were used in a pre-trained CNN model to classify chest CT images as COVID-19 positive/negative.

\section{Screening chest CT images for COVID-19 by multiple pre-trained CNN models}

To fine-tune a pre-trained CNN model, transfer learning is often faster and easier than constructing and training a new $\mathrm{CNN}$ model for a new task. Although a pre-trained $\mathrm{CNN}$ model has already learned a rich set of image features, it can be fine-tuned to learn features specific to a new dataset (i.e., chest CT images from COVID-19 patients in this study). Since a pre-trained CNN model can learn to extract a different feature set, the final CNN model is often more accurate. The starting point for fine tuning deeper layers of pre-trained CNN models used for transfer learning (i.e., VGG-19, Resnet-101, DenseNet-201, Inception-v3, and Inception-ResNet-v2) was training the networks with a new dataset of chest CT images from COVID-19 patients. Figure 7 is a flowchart of the transfer learning procedure used in the $\mathrm{CNN}$ model.

\section{Comparing classification performance among different trained CNN models}

In this study, five independent runs of VGG-19, Resnet-101, DenseNet-201, Inceptionv3, and Inception-ResNet-v2 were performed to classify chest CT images as COVID19 positive or negative by using an algorithm hyperparameter combination obtained by UED. The results recorded for the training set and the validation set included (1) accuracy in each run of the experiment, (2) average accuracy in five independent runs, and (3) standard deviation in accuracy in five independent runs. Accuracy was defined as the proportion of true positive or true negative results for a population.

\section{Selecting the trained CNN models with the high accuracy for use in a COVID19-CNN ensemble model and comparing the classification performance of the ensemble model with that of other trained CNN models}

The high accurate CNN models after training with VGG-19, Resnet-101, DenseNet-201, Inception-v3, and Inception-ResNet-v2 were selected for use in an ensemble model for classifying images in the testing set of chest CT images as COVID-19 positive or negative.

The classification performance of the different trained CNN models was compared in terms of accuracy, precision, recall (i.e., sensitivity), specificity, and $F_{1}$-score values. Precision was assessed by positive predictive value (number of true positives over number of true positives plus number of false positives). Recall (sensitivity) was assessed by

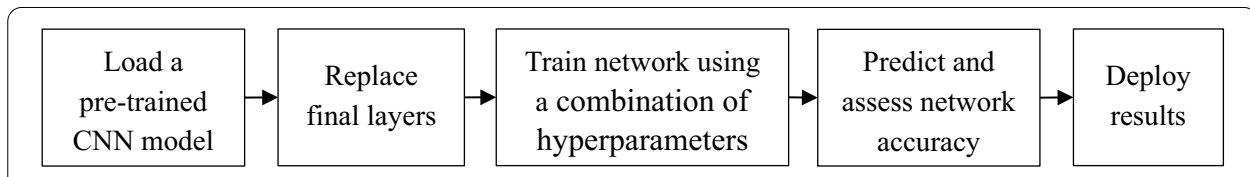

Fig. 7 Flowchart of transfer learning procedure used in the CNN model 
true positive rate (number of true positives over the number of true positives plus the number of false negatives). Specificity was measured by true negative rate (number of true negatives over the number of false positives plus the number of true negatives). The $\mathrm{F}_{1}$-score, a function of precision and recall, was used to measure prediction accuracy when classes were very imbalanced. In information retrieval, precision is a measure of the relevance of results while recall is a measure of the number of truly relevant results returned. The formula for $\mathrm{F}_{1}$-score is $2 \times($ precision $\times$ recall $) /($ precision + recall $)$.

\section{Abbreviations}

COVID-19: Coronavirus disease 2019; CT: Computed tomography; UED: Uniform experimental design; CNN: Convolutional neural network.

\section{Acknowledgements}

Not applicable.

\section{About this supplement}

This article has been published as part of BMC Bioinformatics Volume 22 Supplement 5 2021: Proceedings of the International Conference on Biomedical Engineering Innovation (ICBEI) 2019-2020. The full contents of the supplement are available at https://bmcbioinformatics.biomedcentral.com/articles/supplements/volume-22-supplement-5.

\section{Authors' contributions}

YMC, YJC, and JTT contributed equally to the algorithm and programming design, and theoretical analysis. YMC, WHH, and JTT contributed equally to the quality control and document reviewing. All authors read and approved the final manuscript.

\section{Funding}

Publication costs are funded by the Ministry of Science and Technology, Taiwan, under Grant MOST 109-2221-E-153005-MY3. This work was supported in part by the Ministry of Science and Technology, Taiwan, R.O.C., under Grant Numbers MOST 109-2221-E-037-005, MOST 109-2321-B-037-001, and MOST 109-2221-E-153-005-MY3. This work was also supported in part by the National Pingtung University research project (NPTU-109-020) and the Headquarters of University Advancement and Intelligent Manufacturing Research Center (iMRC) (National Cheng Kung University) sponsored by the Ministry of Education, Taiwan, R.O.C. The funding bodies played no role in any of the following: study design, data collection, analysis, and interpretation; manuscript writing.

\section{Availability of data and materials}

All data obtained and analyzed during this study are included in this article. Chest CT images of COVID-19: https:// github.com/KevinHuRunWen/COVID-19/blob/master/data.zip.

\section{Declarations}

Ethics approval and consent to participate

Not applicable.

\section{Consent for publication}

Not applicable.

\section{Competing interests}

The authors declare that they have no competing interests.

\section{Author details}

${ }^{1}$ School of Nursing, Kaohsiung Medical University, Kaohsiung 807, Taiwan. ${ }^{2}$ Superintendent Office, Kaohsiung Medical University Hospital, Kaohsiung 807, Taiwan. ${ }^{3}$ Management School, National Kaohsiung University of Science and Technology, Kaohsiung 824, Taiwan. ${ }^{4}$ Department of Healthcare Administration and Medical Informatics, Kaohsiung Medical University, Kaohsiung 807, Taiwan. ${ }^{5}$ Department of Medical Research, Kaohsiung Medical University Hospital, Kaohsiung 807, Taiwan. ${ }^{6}$ Department of Computer Science, National Pingtung University, Pingtung 900, Taiwan.

Received: 14 March 2021 Accepted: 16 March 2021

Published online: 08 November 2021

\section{References}

1. Gozes O, Frid-Adar M, Greenspan H, Browning P, Zhang H, Ji W, Bernheim A. Rapid Al development cycle for the coronavirus (COVID-19) pandemic: initial results for automated detection and patient monitoring using deep learning CT image analysis. Submitted to Radiology: Artificial Intelligence; 2020. p. 1-22.

2. Hu R, Ruan G, Xiang S, Huang M, Liang Q, Li J. Automated diagnosis of COVID-19 using deep learning and data augmentation on chest CT. Medrxiv. 2020. https://doi.org/10.1101/2020.04.24.20078998. 
3. Li L, Qin L, Xu Z, Yin Y, Wang X, Kong B, Bai J, Lu Y, Fang Z, Song Q, Cao K, Liu D, Wang G, Xu Q, Fang X, Zhang S, Xia J, Xia J. Artificial intelligence distinguishes COVID-19 from community acquired pneumonia on chest CT. Radiology. 2020. https://doi.org/10.1148/radiol.2020200905.

4. Shan F, Gao Y, Wang J, Shi W, Shi N, Han M, Xue Z, Shi Y. Lung infection quantification of COVID-19 in CT images with deep learning. 2020, p. 1-19. arXiv preprint arXiv:2003.04655.

5. Song Y, Zheng S, Li L, Zhang X, Zhang X, Huang Z, Chen J, Zhao H, Jie Y, Wang R, Chong Y, Shen J, Zha Y, Yang Y. Deep learning enables accurate diagnosis of novel coronavirus (COVID-19) with CT images. MedRxiv. 2020. https://doi. org/10.1101/2020.02.23.20026930.

6. Wang S, Kang B, Ma J, Zeng X, Xiao M, Guo J, Cai M, Yang J, Li Y, Meng X, Xu B. A deep learning algorithm using CT images to screen for corona virus disease (COVID-19). MedRxiv. 2020. https://doi.org/10.1 101/2020.02.14.20023028.

7. Xu X, Jiang X, Ma C, Du P, Li X, Lv S, Yu L, Chen Y, Su J, Lang G, Li Y, Zhao H, Xu K, Ruan L, Wu W.Deep learning system to screen coronavirus disease 2019 pneumonia. 2020. p. 1-29. arXiv preprint arXiv:2002.09334.

8. Yang X, He X, Zhao J, Zhang Y, Zhang S, Xie P. COVID-CT-dataset: a CT scan dataset about COVID-19. 2020. p. 1-14. arXiv preprint arXiv:2003.13865.

9. Yu T, Zhu H. Hyper-parameter optimization: a review of algorithms and applications. 2020. p. 1-56. arXiv preprint arXiv:2003.05689.

10. Kong W, Agarwal PP. Chest imaging appearance of COVID-19 infection. Radiol Cardiothorac Imaging. 2020;2(1):1-22

11. Hu R. Chest CT images of COVID-19. 2020. https://github.com/KevinHuRunWen/COVID-19/blob/master/data.zip

12. Deng J, Dong W, Socher R, Li LJ, Li K, Li FF. ImageNet: a large-scale hierarchical image database. In: Proceedings of the IEEE conference on computer vision and pattern recognition, Florida, USA; 2009. p. 248-55.

13. Russakovsky O, Deng J, Su H, Krause J, Satheesh S, Ma S, Huang Z, Karpathy A, Khosla A, Bernstein M, Berg AC, Li FF. ImageNet large scale visual recognition challenge. Int J Comput Vision. 2015;115:211-52.

14. Simonyan K, Zisserman A. Very deep convolutional networks for large-scale image recognition. In: International conference on learning representations, San Diego, CA, USA; 2015.

15. He K, Zhang X, Ren S, Sun J. Deep residual learning for image recognition. In: Proceedings of the IEEE conference on computer vision and pattern recognition; 2016. p. 770-8.

16. Huang G, Liu Z, Van Der Maaten L, Weinberger KQ. Densely connected convolutional networks. In: Computer vision and pattern recognition; 2017. p. 1-9. arXiv:1608.06993v4.

17. Szegedy C, Vanhoucke V, loffe S, Shlens J, Wojna Z. Rethinking the inception architecture for computer vision. In: Proceedings of the IEEE Conference on Computer Vision and Pattern Recognition; 2016. p. 2818-26.

18. Szegedy C, loffe S, Vanhoucke V, Alemi A. Inception-v4, Inception-ResNet and the impact of residual connections on learning. In: Proceedings of the thirty-first AAAl conference on artificial intelligence; 2017. p. 4278-84.

19. Fang KT. Uniform design and uniform layout. Science Press; 1994.

20. Tsao H, Lee L. Uniform layout implement on Matlab. Stat Decis. 2008;2008(6):144-6.

21. Wang Y, Fang KT. A note on uniform distribution and experimental design. Chin Sci Bull. 1981;26(6):485-9.

\section{Publisher's Note}

Springer Nature remains neutral with regard to jurisdictional claims in published maps and institutional affiliations.

- fast, convenient online submission

- thorough peer review by experienced researchers in your field

- rapid publication on acceptance

- support for research data, including large and complex data types

- gold Open Access which fosters wider collaboration and increased citations

- maximum visibility for your research: over 100M website views per year

At BMC, research is always in progress.

Learn more biomedcentral.com/submissions 